\title{
Aggregated $\alpha$-Synuclein Increases SOD1 Oligomerization in a Mouse Model of Amyotrophic Lateral Sclerosis
}

Yvonne Koch, ${ }^{*}$ Anika M. Helferich, ${ }^{*}$ Petra Steinacker, ${ }^{*}$ Patrick Oeckl, ${ }^{*}$ Paul Walther,${ }^{\dagger}$ Jochen H. Weishaupt, ${ }^{*}$ Karin M. Danzer, ${ }^{*}$ and Markus 0tto*

From the Department of Neurology, * and the Central Facility for Electron Microscopy, ${ }^{\dagger}$ University of Ulm, Ulm, Germany

Accepted for publication April 9, 2016.

Address correspondence to Markus Otto, M.D., Department of Neurology, University of Ulm, Oberer Eselsberg 45, 89081 Ulm, Germany. E-mail: markus.otto@uni-ulm.de.

\begin{abstract}
Aggregation of misfolded disease-related proteins is a hallmark of neurodegenerative diseases. Aggregate propagation accompanying disease progression has been demonstrated for different proteins (eg, for $\alpha$-synuclein). Additional evidence supports aggregate cross-seeding activity for $\alpha$-synuclein. For mutated superoxide dismutase 1 (SOD1), which causes familial amyotrophic lateral sclerosis (ALS), selfpropagation of aggregation and cell-to-cell transmission have been demonstrated in vitro. However, there is a prominent lack of in vivo data concerning aggregation and cross-aggregation processes of SOD1. We analyzed the effect of $\alpha$-synuclein and SOD1 seeds in cell culture using protein fragment complementation assay and intracerebral injection of $\alpha$-synuclein and SOD1 seeds into SOD1 ${ }^{\text {G93A }}$ transgenic ALS mice. Survival of injected mice was determined, and SOD1 aggregates in the facial nuclei were quantified during disease course. We found that $\alpha$-synuclein preformed fibrils increased the oligomerization rate of SOD1 in vivo and in vitro, whereas aggregated SOD1 did not exert any effect in both experimental setups. Notably, survival of ALS mice was not changed after inoculation of preformed fibrils. We conclude that misfolded $\alpha$-synuclein can increase SOD1 aggregation and suppose that $\alpha$-synuclein seeds are transported from the temporal cortex to the facial nuclei. However, unlike other proteins, the further enhancement of a self-aggregation process by additional SOD1 could not be confirmed in our models. (Am J Pathol 2016, 186: 2152-2161; http://dx.doi.org/10.1016/j.ajpath.2016.04.008)
\end{abstract}

Misfolding, accumulation, and deposition of proteins are characteristic for many neurodegenerative disorders [eg, prion diseases, Alzheimer disease (AD), Parkinson disease (PD), Huntington disease, and amyotrophic lateral sclerosis (ALS)]. ${ }^{1}$ Because aggregated abnormally folded proteins were found to contribute to disease progression, a prion-like seeding property of aggregated proteins has been hypothesized. ${ }^{2,3}$ For prion diseases, Alzheimer disease, and Parkinson disease, the progression of disease pathology along certain neural circuits was suggested many years ago ${ }^{4-6}$ and could currently be confirmed by several independent experimental models. ${ }^{7-10}$

A similar mechanism was supposed for ALS. ${ }^{11}$ However, there is a lack of experimental in vivo data, although the most widely used animal model of ALS, the SOD1 $1^{G 93 A}$ model, has existed for two decades. ${ }^{12}$

So far, mutation, oxidation, and posttranslational modification of superoxide dismutase 1 (SOD1) have been found to favor its aggregation. ${ }^{3}$ SOD1 aggregation, seeding, and spreading are controversially discussed as potential diseasecausing mechanisms. ${ }^{3}$ It has been demonstrated in vitro that aggregated SOD1 and tissue homogenate of mutant SODI transgenic mice are able to seed aggregation of SOD1 and can be transmitted between cells. ${ }^{13,14}$ So far, only one study investigated the self-seeding activity of SOD1 in vivo. ${ }^{15}$

Supported by the foundation of the state Baden-Wuerttemberg (BW Stiftung, internal reference D.3830, M.O.), German Federal Ministry for Education and Research (KNDD-FTLDc 01GI1007A, M.O.; MND-net 01GM1103A, M.O. and J.H.W.), the European Union (NADINE 246513, M.O.; SOPHIA 01ED1202A, M.O.; BiomarkAPD 01ED1203F, M.O.; FAIRPARK II 633190, M.O.; PreFront-ALS 01ED1512, M.O.), Boehringer Ingelheim University Ulm Institute (BIU, internal reference D.5009, M.O. and K.M.D.), and Junior Professorship Program Baden-Wuerttemberg (K.M.D.).

Disclosures: None declared. 
A co-presence of $\alpha$-synuclein - the main component of Lewy bodies in Parkinson disease ${ }^{16}$-and SOD1 in the same aggregates has been observed in an ALS patient with SOD1 mutation, ${ }^{17}$ suggesting a cross-seeding activity of $\alpha$-synuclein or SOD1. Cross-seeding describes a process where a misfolded aggregated protein is able to induce the aggregation of a different protein. However, it has not yet been investigated whether other aggregation-prone proteins are able to induce SOD1 oligomerization in vivo.

This could give rise to a different therapeutic strategy: not to target SOD1 aggregates directly but to reduce the trigger for aggregate formation. Because tau and TDP-43 aggregates have been detected after intracerebral injection of $\alpha$-synuclein, a cross-seeding activity of $\alpha$-synuclein has been suggested.

Our aim was to investigate whether $\alpha$-synuclein aggregates are able to induce aggregation and spreading of SOD1. Therefore, we used a SOD1 protein fragment complementation assay (PCA) to study SOD1 aggregation properties in cell culture in the presence of $\alpha$-synuclein preformed fibrils (PFFs) and brainstem homogenate containing SOD1 ${ }^{\mathrm{G} 93 \mathrm{~A}}$ aggregates. In vivo, we investigated $\mathrm{SOD} 1^{\mathrm{G} 93 \mathrm{~A}}$ aggregation after injection of $\alpha$-synuclein PFFs or brainstem homogenate containing SOD $1{ }^{\mathrm{G} 93 \mathrm{~A}}$ aggregates into the temporal cortex of $S O D 1^{G 93 A}$ mice. The mouse model we used expresses a high copy number of the transgene encoding the human ALS causing $S O D 1^{G 93 A}$ mutation. It has symptoms and pathologic alterations reminiscent of human ALS cases with SOD1 mutation. ${ }^{18} \mathrm{We}$ characterized the mice by determining disease onset, end stage, and disease time course. Furthermore, we investigated SOD1 ${ }^{\mathrm{G} 93 \mathrm{~A}}$ and $\alpha$-synuclein inclusions in the facial nerve nuclei, which are affected brain regions in ALS and distantly localized from injection site.

\section{Materials and Methods}

\section{Preparation of Aggregated Proteins}

For intracerebral injection of SOD $1^{\mathrm{G} 93 \mathrm{~A}}$ aggregates, brainstem tissue from transgenic B6SJL-Tg(SOD1*G93A)1Gur/J mice (Jackson Laboratory, Ben Harbor, ME) at terminal disease stage was sonicated in phosphate-buffered saline (PBS). As control, brainstem tissue from nontransgenic age-matched littermates was used. After centrifugation for 15 minutes at $14,000 \times g$, protein concentration of the supernatant was determined using a bicinchonic acid assay (Thermo Scientific, Rockford, IL) and adjusted to $5 \mu \mathrm{g} / \mu \mathrm{L}$ of protein. The PFFs were generated from recombinant human $\alpha$-synuclein protein with a purity of $>95 \%$ (a gift from Boehringer Ingelheim Pharma GmbH \& Ko. KG, Biberach an der Riss, Germany). $\alpha$-Synuclein was reconstituted in fibril assembly buffer (50 $\mathrm{mmol} / \mathrm{L}$ Tris, $100 \mathrm{mmol} / \mathrm{L} \mathrm{NaCl}, \mathrm{pH} 7.0$ ) at a concentration of $5 \mu \mathrm{g} / \mu \mathrm{L}$ and $200 \mu \mathrm{L}$ per tube of the solution and was incubated in an orbital shaker at $37^{\circ} \mathrm{C}$ and $1000 \mathrm{rpm}$ for 5 days as previously described. ${ }^{19}$ Both aggregate-containing preparations were aliquoted and stored at $-80^{\circ} \mathrm{C}$.

\section{Nonreducing SDS-PAGE}

A total of $20 \mu \mathrm{g}$ of brainstem homogenate or $5 \mu \mathrm{g}$ of PFFs were separated on $4 \%$ to $20 \%$ Mini-PROTEAN TGX Gels (Bio-Rad, Hercules, CA) under nonreducing conditions as described previously. ${ }^{20}$ Proteins were transferred to a polyvinylidene difluoride membrane and probed with antibodies against SOD1 (ab16831, Abcam, Cambridge, UK), $\alpha$-synuclein (D37A6, Cell Signaling Technology, Cambridge, UK), or hypoxanthine-guanine phosphoribosyltransferase (ab109021; Abcam). Secondary anti-rabbit antibody labeled with horseradish peroxidase (Dianova, Hamburg, Germany) was used to convert Luminata forte substrate (Merck Millipore, Darmstadt, Germany). Chemiluminescence signal was detected using the ImageQuant LAS 4000 system (GE Healthcare Life Sciences, Chalfont St Giles, UK).

\section{Protein Aggregate Filtration Assay}

Five micrograms of brainstem homogenate was passed through a $0.2-\mu \mathrm{m}$ pore size nitrocellulose membrane using a Dot Blot SF Microfiltration apparatus (Bio-Rad). Hereby only aggregated SOD1 but not the dimeric form is trapped on the membrane and can be immunodetected as described above. ${ }^{21,22}$ Equal protein amounts on the membrane were demonstrated by Ponceau S staining.

\section{Electron Microscopy}

Untreated recombinant $\alpha$-synuclein and PFF solution were diluted 1:20 in PBS, and $5 \mu \mathrm{L}$ each was incubated for 1 minute on a formvare-coated square 300 mesh copper electron microscopy sample grid (Plano, Wetzlar, Germany), which was dried afterward, incubated for 1 minute with $2 \%$ uranyl acetate, and then dried again. Samples were analyzed using an EM10 (Carl Zeiss, Jena, Germany).

\section{Protein Complementation Assay}

In the fusion constructs $\alpha$-synuclein- Gaussia luciferase (hGluc1) (S1) and $\alpha$-synuclein-hGluc2 (S2), ${ }^{23} \alpha$-synuclein was replaced by SOD1 or SOD $1^{\mathrm{G} 93 \mathrm{~A}}$ cDNA to generate SOD1-hGluc1 (SOD1-L1) and SOD1-hGluc2 (SOD1-L2) or SOD1 ${ }^{\mathrm{G} 93 \mathrm{~A}}$-hGluc1 (G93ASOD1-L1) and SOD1 ${ }^{\mathrm{G} 93 \mathrm{~A}}$ hGluc2 (G93ASOD1-L2). As a control, plasmids L1 and L2 that contained hGluc halves only were used. $\mathrm{H} 4$ cells in 96-well plates were co-transfected with equimolar ratios of SOD1-L1 + SOD1-L2 or G93ASOD1-L1 + G93ASOD1$\mathrm{L} 2$ or $\mathrm{L} 1+\mathrm{L} 2$ or in case of mock without plasmid constructs using Fugene6 transfection reagent (Promega, Fitchburg, WI) according to the manufacturer's instructions. Twenty-four hours after transfection, culture medium was replaced with $100 \mu \mathrm{L}$ of serum- and phenol red-free OptiMEM (Life Technologies, Carlsbad, CA) containing $7 \mu \mathrm{mol} / \mathrm{L} \quad(96 \mathrm{ng} / \mu \mathrm{L}) \quad \alpha$-synuclein PFFs, brainstem 
homogenate from transgenic SOD $1^{\mathrm{G} 93 \mathrm{~A}}$ mice, or nontransgenic littermates containing $96 \mathrm{ng} / \mu \mathrm{L}$ protein or the same volume of the corresponding buffer. After 24 hours, $80 \mu \mathrm{L}$ of the culture medium was transferred to a new 96well plate (referred to as conditioned medium). Remaining medium was aspirated, and $100 \mu \mathrm{L}$ of serum- and phenol red-free OptiMEM was added. Luciferase activity of conditioned medium and cells was measured using a luminometer (Victor3 multilabel plate reader; PerkinElmer, Waltham, MA) after addition of cell permeable coelenterazine $(20 \mu \mathrm{mol} / \mathrm{L}$; Nanolight, Pinetop, AZ).

\section{Mouse Breeding and Genotyping}

All animal experiments were performed in accordance with national laws and guidelines and were approved by state authorities.

Male mice carrying the human $S O D 1^{G 93 A}$ transgene [B6SJL- $\operatorname{Tg}(\mathrm{SOD} 1 * \mathrm{G} 93 \mathrm{~A}) 1 \mathrm{Gur} / \mathrm{J}]$ were obtained from the Jackson Laboratory and cross-bred with female C57BL/6 mice. Offspring was analyzed for the presence of the $S O D 1^{G 93 A}$ transgene as previously described. ${ }^{24}$

To ensure an equal amount of $S O D 1^{G 93 A}$ transgene within the experimental groups, the difference in threshold cycle $(\triangle \mathrm{CT})$ of SODI and IL2 amplification of symptomatic mice was analyzed. Therefore, real-time PCR assays were performed using the LightCycler 480 instrument (Roche Diagnostics, Basel, Switzerland) with primers and hydrolysis probes designed by the Roche Universal Probe Library (UPL) system. The following primer sequences and UPLs were used for quantitative real-time PCR: $S O D 1^{G 93 A}$ : 5'-AGATGACTTGGGCAAAGGTG-3', 5'-TATTGGGCGATCCCAATTAS-3', UPL $11, I l 2: 5^{\prime}$-TCCTGTGAACTCTATGTTAATCAGTGT-3', 5'-TTTTCCCACAAGCCATCTTT-3', UPL 91. We detected no difference regarding $\Delta \mathrm{CT}$ values among the differently inoculated mice. The $\Delta \mathrm{CT}$ levels matched published $\Delta \mathrm{CT}$ values of the high-copy SOD $1^{\mathrm{G} 93 \mathrm{~A}}$ mouse model. ${ }^{25}$ Therefore, we can exclude an influence of divergent copy numbers on disease course or survival.

\section{Intracerebral Injection}

Forty-day-old $S O D 1^{G 93 A}$ transgenic mice were anesthetized with an i.p. injection of $100 \mathrm{mg} / \mathrm{kg}$ of ketamine hydrochloride and $16 \mathrm{mg} / \mathrm{kg}$ of xylazine and stereotaxically injected with $5 \mu \mathrm{g}$ of brainstem homogenate containing SOD $1^{\mathrm{G} 93 \mathrm{~A}}$ aggregates (male mice), $5 \mu \mathrm{g}$ of $\alpha$-synuclein PFFs (female mice), or $1 \mu \mathrm{L}$ of $0.9 \%$ sodium chloride solution (saline, male and female mice). Mice were injected in the right hemisphere $2 \mathrm{~mm}$ posterior and $3.7 \mathrm{~mm}$ lateral relative to bregma with a puncture depth of $3.3 \mathrm{~mm}$ with an angle of $20^{\circ}$ into the insular cortex using a Hamilton syringe at a rate of $0.5 \mu \mathrm{L}$ per minute. The needle remained at the target site for 2 minutes. After recovery from surgery, mice were monitored regularly and sacrificed $1,7,40$, or 70 days post injection (dpi) or when they reached disease end stage (Table 1).

\section{Tissue Preparation}

Mice were sacrificed using an overdose thiopental and transcardially perfused with $10 \mathrm{~mL}$ of PBS followed by $10 \%$ neutral buffered formalin solution (Sigma-Aldrich, St. Louis, MO). The brains were removed and postfixed in $10 \%$ neutral buffered formalin solution. Tissue was paraffin embedded and cut into $7-\mu \mathrm{m}$ coronal sections.

\section{Histologic Analysis of SOD $1^{\mathrm{G} 93 \mathrm{~A}}$ Aggregation and $\alpha$-Synuclein}

To test whether B6SJL-Tg(SOD1*G93A)1Gur/J mice had $\mathrm{SOD} 1^{\mathrm{G} 93 \mathrm{~A}}$-positive aggregates in the brainstem, $12-\mu \mathrm{m}$ cryoconserved sections were fixed for 10 minutes in $10 \%$ neutral buffered formalin solution. Afterward, sections were stained in $1 \%$ Thioflavin S in PBS for 5 minutes and washed twice in ethanol and twice in PBS. Sections were blocked with 5\% goat serum in PBS for 1 hour and incubated with anti-human SOD1 antibody (ab52950, diluted 1:50; Abcam) at $4^{\circ} \mathrm{C}$ overnight. A secondary antibody labeled with Alexa 546 dye (Life Technologies) was used for detection of SOD1. Sections were mounted in Mowiol containing $25 \mathrm{mg} / \mathrm{mL}$ of DABCO [1,4diazabicyclo(2.2.2)octane] as an antifading reagent and $1 \mu \mathrm{g} /$ $\mathrm{mL}$ of Hoechst 33342 for nuclear staining. Sections were analyzed using a Axiovert 200 fluorescence microscope and AxioVision software version 4.8.2.0 (Carl Zeiss).

For immunohistochemical detection of SOD $1^{\mathrm{G} 93 \mathrm{~A}}$ aggregates or $\alpha$-synuclein, paraffin sections were rehydrated and antigen retrieval was achieved by boiling the sections for 5 minutes in water (SOD1 ${ }^{\mathrm{G} 93 \mathrm{~A}}$ ) or for 20 minutes in $10 \mathrm{mmol} / \mathrm{L}$ citrate buffer $(\mathrm{pH} 6)(\alpha$-synuclein) at $800 \mathrm{~W}$ in the microwave. Endogenous peroxidases were blocked with 3\% hydrogen peroxide in PBS, and the cell membrane

Table 1 Characteristics of Experimental Groups for the Inoculation Study

\begin{tabular}{|c|c|c|c|c|c|c|c|}
\hline \multirow[b]{2}{*}{ Inoculum } & \multicolumn{5}{|c|}{ No. of mice at time of brain dissection } & \multirow[b]{2}{*}{ Sex } & \multirow{2}{*}{$\begin{array}{l}\text { Age at } \\
\text { inoculation } \\
\text { days }\end{array}$} \\
\hline & $1 \mathrm{dpi}$ & $7 \mathrm{dpi}$ & 40 dpi & 70 dpi & $\begin{array}{l}\text { Disease } \\
\text { end stage }\end{array}$ & & \\
\hline Brainstem homogenate containing aggregated SOD1 & 5 & 5 & 5 & 5 & 10 & Male & 40 \\
\hline$\alpha$-Synuclein PFFs & 5 & 5 & 5 & 5 & 10 & Female & 40 \\
\hline Saline & 5 & 5 & 5 & 5 & 10 & Male and female & 40 \\
\hline
\end{tabular}

dpi, days post injection; PFF, preformed fibril. 
was permeabilized using $0.2 \%\left(\mathrm{SOD} 1^{\mathrm{G} 93 \mathrm{~A}}\right)$ or $0.5 \%$ $(\alpha$-synuclein) Triton X-100 in PBS. Sections were blocked with $5 \%$ goat serum $\left(\mathrm{SOD} 1^{\mathrm{G} 93 \mathrm{~A}}\right)$ or $2 \%$ goat serum $/ 2 \%$ bovine serum albumin ( $\alpha$-synuclein) in PBS and incubated at $4^{\circ} \mathrm{C}$ overnight with anti-human SOD1 antibody (ab52950; Abcam) diluted 1:500 or anti- $\alpha$-synuclein antibody (D37A6; Cell Signaling Technology) diluted 1:150. After three washes with PBS, sections were incubated with secondary antibody labeled with horseradish peroxidase (Dianova) and developed with DAB (Sigma-Aldrich). Sections were counterstained with $0.1 \%$ cresyl violet and mounted with Entellan.

Sections stained for SOD1 ${ }^{\mathrm{G} 93 \mathrm{~A}}$ were digitalized using a Mirax Desk Scanner (Carl Zeiss Microimaging, Göttingen, Germany) and analyzed at 10-fold digital magnification using ImageJ version 1.47v (NIH, Bethesda, MD; http:// imagej.nih.gov/ij). Cresyl violet-positive, triangle-shaped cells with $>15-\mu \mathrm{m}$ diameter were classified as motor neurons. SOD1-positive structures with $>2-\mu \mathrm{m}$ diameter were considered as $\mathrm{SOD} 1^{\mathrm{G} 93 \mathrm{~A}}$ aggregates and were counted within the region of the facial nuclei. The numbers of motor neurons or aggregates of every 20th section showing the facial nuclei (five slices per animal) were added together and normalized to the area examined. Calculation was performed for both and single hemispheres individually. Data presented in this article represent the values from analysis of both hemispheres if not indicated differently.

Immunoreactivity and distribution of $\alpha$-synuclein were analyzed in the facial nuclei using a brightfield microscope with 40-fold magnification (Axioskop 2 plus, Carl Zeiss).

The evaluator (Y.K.) was blinded regarding the age of mice and the injected substance.

\section{Disease Staging}

Disease staging was conducted as previously described. ${ }^{24}$ After surgery, body weight was measured once a week. With the appearance of first disease symptoms, mice were weighed daily to monitor their health and disease progression.

\section{Statistical Analysis}

All statistical analyses were performed using GraphPad Prism software version 5.00 (GraphPad Software Inc., La Jolla, CA). The PCA data were analyzed for statistical difference using a two-tailed, unpaired $t$-test. Data from mouse experiments were analyzed using the Kruskal-Wallis test with a Dunn posttest. Survival was analyzed by $\log$ rank test. $P \leq 0.05$ was regarded as significant. Data are presented as means \pm SEM.

\section{Results}

For seeding and cross-seeding experiments, we proved that our preparations contained defined SOD $1^{\mathrm{G} 93 \mathrm{~A}}$ aggregates and $\alpha$ synuclein PFFs. Immunofluorescent analysis of brainstem tissue of end-stage $S O D 1^{G 93 A}$ transgenic mice-which was used for additional experiments later-revealed mostly
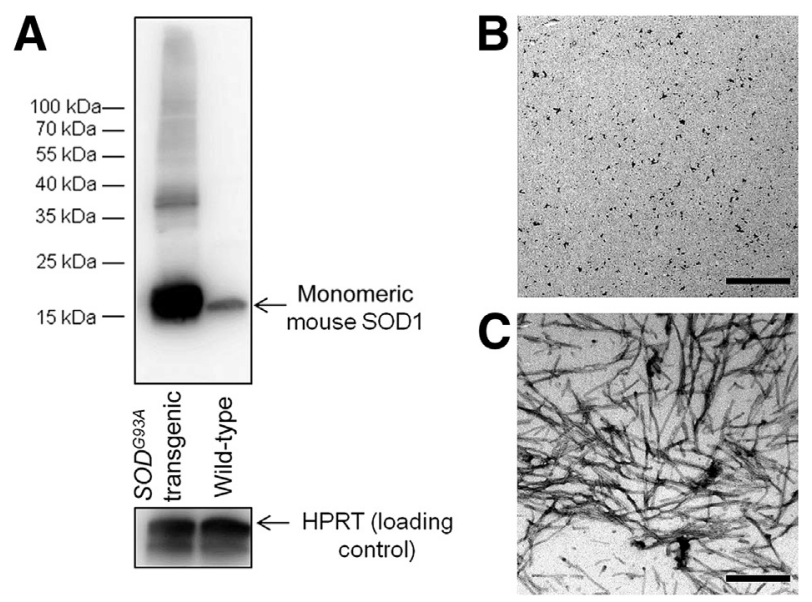

Figure 1 SOD1 and $\alpha$-synuclein preparations contain protein aggregates. A: Immunoblot of tissue homogenate from end-stage $S O D 1^{G 93 A}$ transgenic mice and wild-type littermates using SOD1 antibody after nonreducing SDS-PAGE. SOD1 species $>100 \mathrm{kDa}$ can be detected only in the transgenic mouse. Hypoxanthine-guanine phosphoribosyltransferase (HPRT) was visualized as loading control. Electron micrograph after negative staining of untreated $\alpha$-synuclein (B) and $\alpha$-synuclein preformed fibrils (C). Scale bars $=600 \mathrm{~nm}$ (B and C).

roundish or oval structures that tested positive for Thioflavin $\mathrm{S}$ and human SOD1. Those structures could not be detected in a nontransgenic littermate (Supplemental Figure S1A).

To investigate whether SOD1 ${ }^{\mathrm{G} 93 \mathrm{~A}}$ aggregates were still present after homogenization of the brainstem, nonreducing SDS-PAGE (Figure 1A) and protein aggregate filtration assay (Supplemental Figure S1B) were conducted. Using anti-SOD1 antibody, high-molecular SOD1 species were detected in $S O D 1^{G 93 A}$ transgenic but not in wild-type mice, indicating the presence of aggregated SOD ${ }^{\mathrm{G} 93 \mathrm{~A}}$.

To control $\alpha$-synuclein PFF preparation, negative stain electron microscopy was performed. Untreated recombinant protein exhibited a dot-like roundish morphology (Figure 1B), whereas PFFs had a fibrillar morphology. Fibrils had a length between 50 and $500 \mu \mathrm{m}$ and assembled to a net-like structure (Figure 1C). In addition, the presence of high-molecular $\alpha$-synuclein species within PFF preparation was confirmed by nonreducing SDS-PAGE (Supplemental Figure S1C).

These preparations were used to determine their influence on SOD1 aggregation in vitro. For this, we used the hGluc PCA which has already been used to analyze $\alpha$-synuclein oligomerization. ${ }^{23,26}$ Human $\mathrm{H} 4$ neuroglioma cells were cotransfected with plasmid constructs encoding for SOD1-L1 and SOD1-L2. Because of SOD1 interaction and oligomerization, the two hGluc fragments are brought together and hGluc enzymatic activity is reconstituted. Cells were incubated with $\alpha$-synuclein PFFs, transgenic or wild-type mouse brainstem homogenate or corresponding buffers for 24 hours, followed by luciferase activity measurement.

Incubation with $\alpha$-synuclein PFFs increased luciferase activity 1.6-fold in living cells transfected with SOD1-L1 and SOD1-L2 $(P=0.0001)$ (Figure $2 \mathrm{~A})$ and their conditioned medium $(P<0.0001)$ (Figure 2D). In the medium of cells 


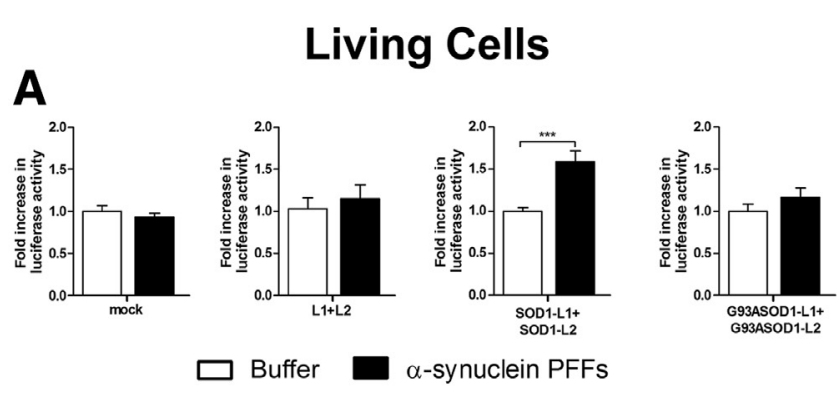

B

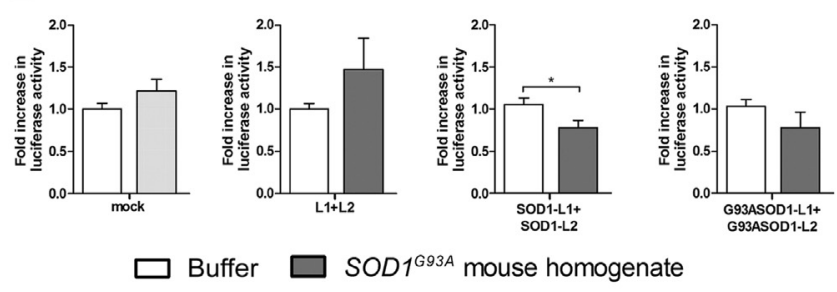

C
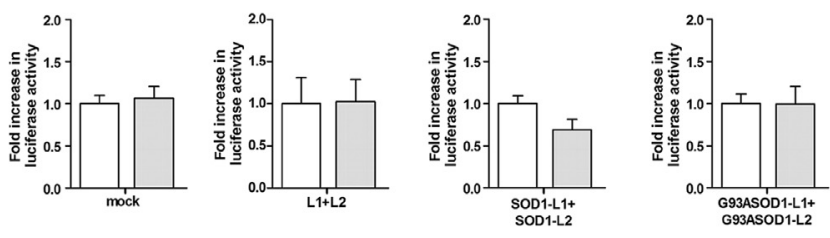

Buffer $\square$ Wild-type mouse homogenate

\section{Conditioned Medium}

D
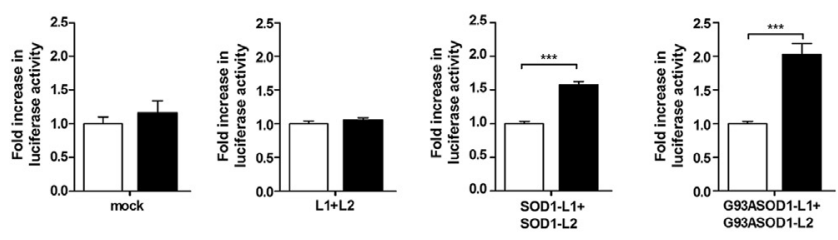

Buffer

$\alpha$-synuclein PFFs

E
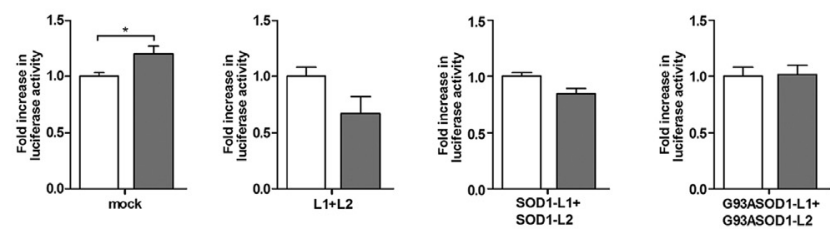

Buffer

SOD $1^{\text {G93A }}$ mouse homogenate

$\mathbf{F}$
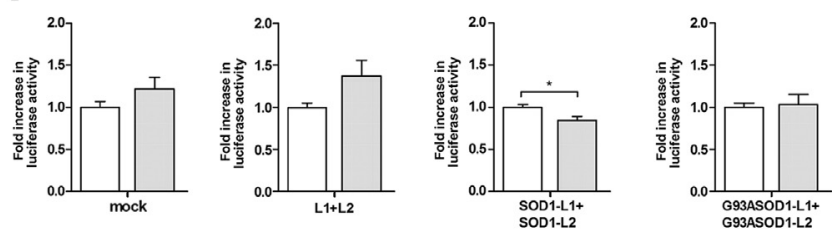

Buffer $\square$ Wild-type mouse homogenate transfected with G93ASOD1-L1 + G93ASOD1-L2, a twofold increase in luciferase activity was also observed $(P<0.0001)$ (Figure 2D). In contrast, $\alpha$-synuclein PFFs did not influence the luciferase activity in mock or L1 and L2 transfected cells (Figure 2A) or their conditioned medium (Figure 2D), suggesting that $\alpha$-synuclein PFFs increase the oligomerization of SOD1 and SOD $1^{\mathrm{G} 93 \mathrm{~A}}$.

Incubation with mouse brainstem homogenate from $S O D 1^{G 93 A}$ transgenic mice did not influence luciferase activity in cells (Figure 2B) and their conditioned medium (Figure 2E) except in cells transfected with SOD1$\mathrm{L} 1+$ SOD1-L2. SOD1 ${ }^{G 93 A}$ mouse brain homogenate led to a decrease of approximately $20 \%(P=0.0258)$ in luciferase activity of cells transfected with SOD1L1 + SOD1-L2 compared with cells treated with the corresponding buffer (Figure 2B). In the conditioned medium of mock transfected cells, a $20 \%$ increase in luciferase activity was detected after incubation with brainstem homogenate of transgenic mice (Figure 2E). Incubation with brainstem homogenate of a wild-type littermate did not result in any changes in luciferase activity in living cells (Figure 2C). In conditioned medium of cells transfected with SOD1-L1 + SOD1-L2, wild-type brainstem homogenate led to a $15 \%$ decrease of luciferase activity $(P=0.02)$ (Figure 2F). These data indicate that $\alpha$-synuclein but not SOD1 is able to increase SOD1 oligomerization.

To determine whether $\alpha$-synuclein PFFs or brainstem homogenate containing $\mathrm{SOD} 1^{\mathrm{G} 93 \mathrm{~A}}$ aggregates influence SOD1 aggregation in vivo, we inoculated $S O D 1^{G 93 A}$ transgenic mice with $\alpha$-synuclein PFFs or brainstem homogenate containing SOD $1^{\mathrm{G} 93 \mathrm{~A}}$ aggregates into the temporal cortex and investigated the number of $\mathrm{SOD} 1^{\mathrm{G} 93 \mathrm{~A}}$ positive aggregates in the facial nerve nuclei at different time points by $\mathrm{SOD} 1^{\mathrm{G} 93 \mathrm{~A}}$ immunohistochemistry. Representative sections of the facial nerve nuclei used for quantification of motor neurons and aggregates are shown in Figure 3.

During the disease course, we found an increasing number of SOD $1^{\mathrm{G} 93 \mathrm{~A}}$ aggregates per square millimeter until 40 dpi, when aggregate counts reached their maximum in all groups. From 40 dpi until disease end stage, $\mathrm{SOD} 1^{\mathrm{G} 93 \mathrm{~A}}$ aggregate counts decreased but a more intense surrounding staining for SOD1 ${ }^{\mathrm{G} 93 \mathrm{~A}}$ could be observed (Figure 3 and

Figure $2 \alpha$-Synuclein but not SOD1 aggregates increase luciferase activity in protein fragment complementation assay. Luciferase activity measurement of mock/L1 + L2/SOD1-Luc1 + SOD1-Luc2/G93ASOD1Luc1 + G93AS0D1-Luc2 transfected $\mathrm{H} 4$ cells and conditioned medium after incubation with $7 \mu \mathrm{mol} / \mathrm{L}$ a-synuclein preformed fibrils (PFFs) (A and D), brainstem homogenate from SOD ${ }^{\mathrm{Gg}}{ }^{\mathrm{AA}}$ transgenic (B and $\left.\mathbf{E}\right)$, or wild-type mice and corresponding buffer for 24 hours (C and $\mathbf{F})$. Incubation with PFFs increases luciferase activity significantly in SOD1-L1 + SOD1-L2 transfected cells (A) and conditioned medium from SOD1-L1 + S0D1-L2/G93ASOD1L1 + G93AS0D1-L2 transfected cells (D). Data are from three to six independent experiments. Data are expressed as means + SEM. $n=9$ to 18 . ${ }^{*} P<0.05,{ }^{* * *} P<0.001$ (two-tailed, unpaired $t$-test). 

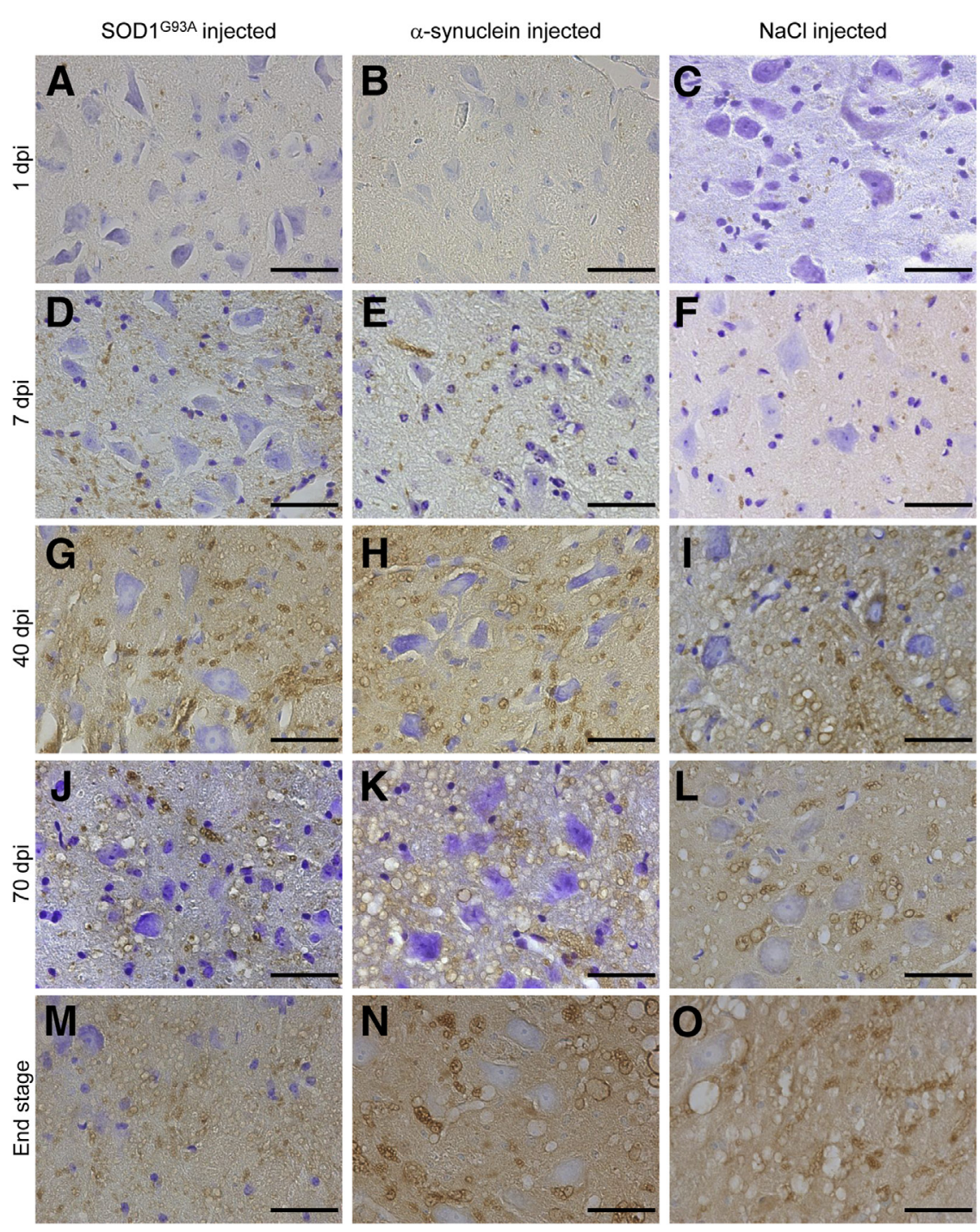

Figure 3 Representative images of sections stained for SOD1 in the area of the facial nucleus 1 $(\mathbf{A}-\mathbf{C}), 7$ (D-F), $40(\mathbf{G}-\mathbf{I})$, and 70 (J-L) days post injection (dpi) and at disease end stage $(\mathbf{M}-\mathbf{0})$. Scale bars $=50 \mu \mathrm{m}(\mathbf{A}-\mathbf{0})$.

Figure 4A). When we compared the different groups at certain time points, we found a significant difference in the number of aggregates per square millimeter between $\alpha$-synuclein- and saline-injected mice at $70 \mathrm{dpi}$ $(509.2 \pm 86.11$ versus 308.3 $\pm 91.24, P=0.03)$ (Figure 4A). However, a trend toward higher numbers of SOD 1 G93A aggregates per square millimeter in $\alpha$-synuclein- compared with $\mathrm{NaCl}$-injected mice could also be detected at $40 \mathrm{dpi}$ $(729.5 \pm 65.88$ versus $526.9 \pm 156.2, P=0.06)$ and disease end stage $(400.4 \pm 68.5$ versus $279.2 \pm 39.22$, $P=0.11$ ). At 1 and $7 \mathrm{dpi}$, no differences between the groups could be detected. For $S O D 1^{G 93 A}$ mice injected with SOD ${ }^{\mathrm{G} 93 \mathrm{~A}}$ aggregate containing brainstem homogenate, we could not find any differences in SOD $1^{\mathrm{G} 93 \mathrm{~A}}$ aggregate numbers, suggesting that SOD1 ${ }^{\mathrm{G} 93 \mathrm{~A}}$ aggregates do not influence $\mathrm{SOD} 1{ }^{\mathrm{G} 93 \mathrm{~A}}$ aggregation under these in vivo conditions (Figure 4A).

In addition, we compared the diameter of SOD1 ${ }^{\mathrm{G} 93 \mathrm{~A}}$ aggregates of the groups at different time points. During the disease course, the aggregate diameter significantly $(P=0.03)$ increased in $\alpha$-synuclein-injected mice between 40 dpi and disease end stage (Figure 4B). The aggregate diameter tended to increase in all experimental groups. The different groups had similarly sized aggregates at certain time points, indicating that higher SOD $1^{\text {G93A }}$ aggregate counts in $\alpha$-synuclein-injected mice are not attributable to the differences in the size of the aggregates.

Because the number of motor neurons in $S O D 1^{G 93 A}$ mice correlates with severity of disease symptoms, we evaluated the number of motor neurons in the facial motor nuclei, one of the affected brain regions in ALS. We found a significant decrease in the number of motor neurons per square millimeter in all experimental groups between $1 \mathrm{dpi}$ and disease end stage, but no significant difference between the groups at any time point was detected (Figure 4C).

We compared aggregate counts, motor neuron counts, and $\alpha$-synuclein staining in the facial nuclei from the left and right hemisphere to evaluate possible changes after unilateral injection of aggregated SOD $1^{\mathrm{G} 93 \mathrm{~A}}$ or $\alpha$-synuclein PFFs. No differences between the hemispheres were detected (Figure 4D). 

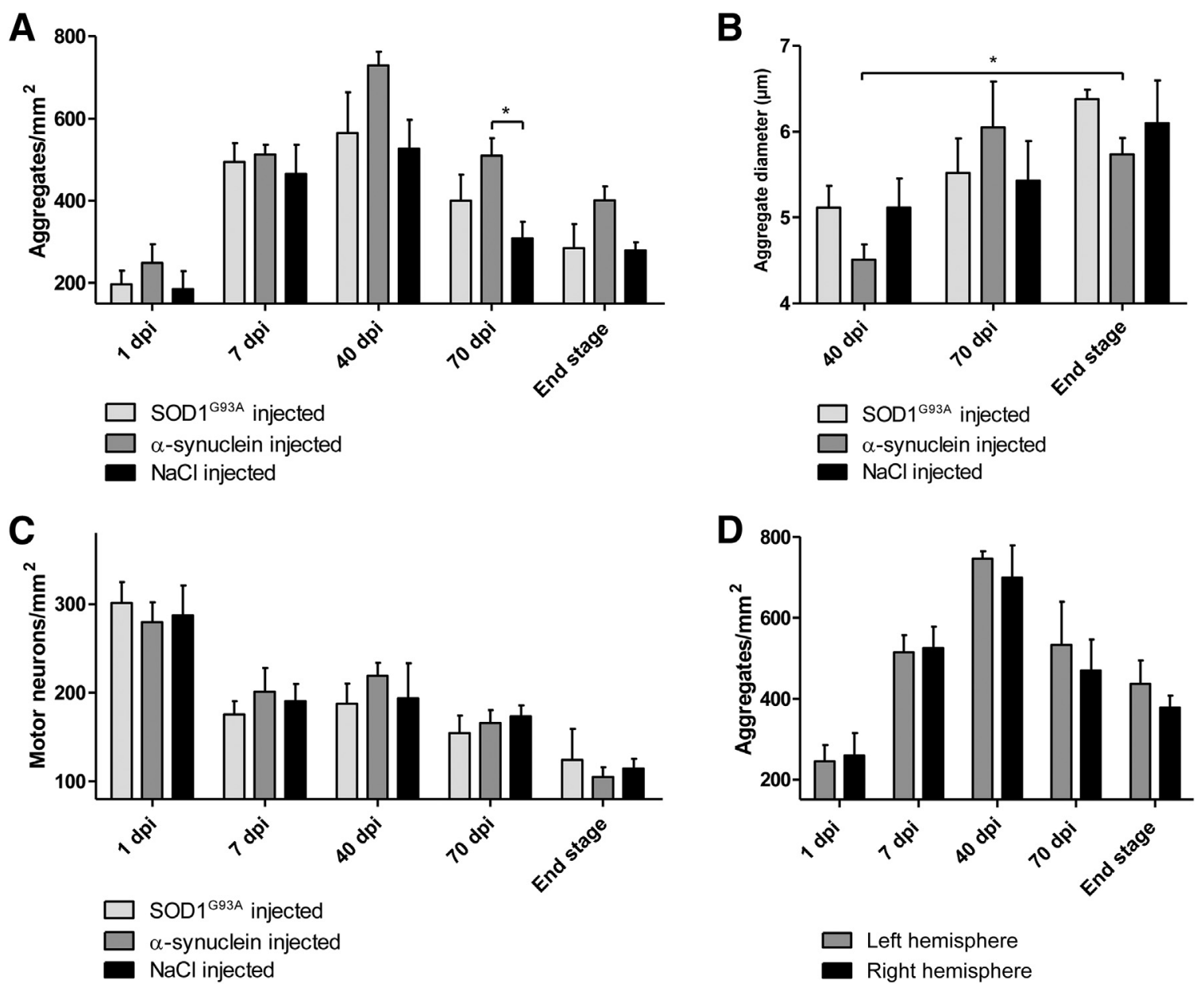

Figure 4 Elevated numbers of SOD1 aggregates after injection of $\alpha$-synuclein preformed fibrils into the temporal cortex of SOD1 ${ }^{\text {G93A }}$ mice, whereas aggregate size and motor neuron count do not differ. A: A significantly higher number of SOD1 ${ }^{\text {G93A }}$ aggregates can be detected in the facial nucleus 70 days post infection (dpi) between $\alpha$-synuclein- and saline-injected mice. A trend toward higher aggregate numbers in $\alpha$-synuclein injected mice can be seen 40 dpi and at disease end stage. B: A significant increase in $\mathrm{SOD}_{1}{ }^{\mathrm{G9} 3 \mathrm{~A}}$ aggregate diameter can be detected in $\alpha$-synuclein-injected mice over time. A trend toward bigger aggregate diameter can be observed in all experimental groups. No differences between the groups could be detected at any time point investigated. C: The number of motor neurons detected by cresyl violet staining decreases during disease course but does not differ between experimental groups. D: In $\alpha$-synuclein-injected mice, no difference between the left and right hemispheres regarding SOD $1^{\text {G93A }}$ aggregate counts can be detected. $n=4$ to 5 . ${ }^{*} P<0.05$ (Kruskal-Wallis test and Dunn posttest).

Taken together, using immunohistochemistry, we found that $\alpha$-synuclein PFFs are able to form SOD1 ${ }^{\mathrm{G} 93 \mathrm{~A}}$ aggregates in vivo. Despite increased numbers of SOD1 ${ }^{\text {G93A }}$ aggregates in both hemispheres of the brain, disease-related pathologic changes (ie, motor neuron counts) were not influenced in $S O D 1^{G 93 A}$ mice.

Because aggregated $\alpha$-synuclein induces its own aggregation in wild-type mice in a prion-like manner and spreads by so far unknown mechanisms along neural circuits after intracerebral inoculation, ${ }^{7,27}$ we also investigated $\alpha$-synuclein levels in the facial nuclei of the mice. $\alpha$-Synuclein immunoreactivity was similar in the differently injected mice except for $70 \mathrm{dpi}$. At this time point, $\alpha$-synuclein staining was much higher in $\alpha$-synuclein PFF-injected animals compared with aggregated SOD1- or NaCl-injected mice (Figure 5).

To evaluate the effect of increased SOD ${ }^{\mathrm{G} 93 \mathrm{~A}}$ aggregation in $\alpha$-synuclein-injected mice, we analyzed the time course of symptoms and survival of the mice. Only sex-specific differences were found. Weight development (Figure 6A), age when first symptoms appeared (Figure 6B), first pareses of the hind limbs appeared (Figure 6C), or overall survival of the mice (Figure 6, D and E) was not altered after injection of SOD1 aggregates or $\alpha$-synuclein PFFs. In addition, no significant differences in the duration of symptomatic phase (early and late disease phase) was observed (data not shown).

\section{Discussion}

Since Braak and Braak $^{5}$ reported a constant spreading pattern of pathologic changes in Alzheimer disease, the idea of aggregate self-propagation arose over time not only for prion diseases but also for other neurodegenerative diseases. Recently, the principle of aggregate cross-seeding has also been suggested for aggregation-prone proteins. ${ }^{7,28,29}$ 
SOD1 ${ }^{\mathrm{G} 93 \mathrm{~A}}$ injected

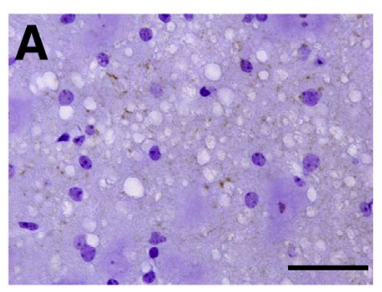

$\alpha$-synuclein injected

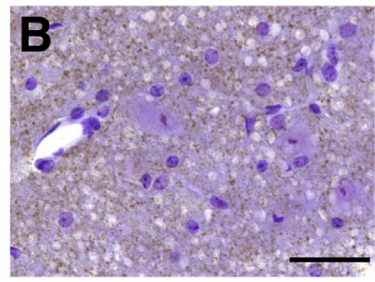

$\mathrm{NaCl}$ injected

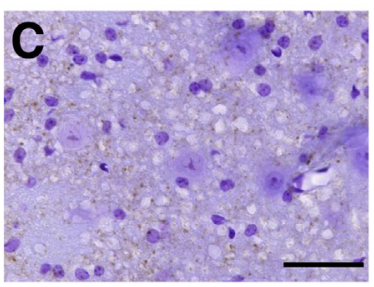

Figure 5 Representative images of sections stained for $\alpha$-synuclein showing the facial nucleus of mice 70 days post injection of brainstem homogenate from SOD1 ${ }^{\mathrm{G} 93 \mathrm{~A}}$ transgenic mice (A), $\alpha$-synuclein PFFs (B), or saline (C). Scale bars $=$ $50 \mu \mathrm{m}(\mathbf{A}-\mathbf{C})$.
In our cell culture model, we found that SOD1 has a higher oligomerization rate in the presence of $\alpha$-synuclein PFFs. In vivo we were able to confirm this effect because a higher amount of SOD $1^{\mathrm{G} 93 \mathrm{~A}}$ accumulation after $\alpha$-synuclein PFF inoculation was observed.

Using PCA, we found that $\alpha$-synuclein PFFs increase oligomerization of SOD1. Enhanced oligomerization of wild-type SOD1 was detected in living cells and conditioned medium, whereas mutant SOD1 oligomerization was increased in conditioned medium only. PFF treatment might result in a fast secretion of mutant SOD1 because an additional secretion mechanism for mutant SOD1 is known. ${ }^{30}$ Furthermore, a higher level of dimer formation in untreated SOD $1{ }^{\mathrm{G} 93 \mathrm{~A}}$ transfected cells could conceal the effect elicited by $\alpha$-synuclein on SOD $1^{\mathrm{G} 93 \mathrm{~A}}$ oligomerization inside the cells. A similar increase in SOD1 oligomerization has been found in the presence of untreated recombinant $\alpha$-synuclein in a recent publication, which also reports the specificity of the SOD1- $\alpha$-synuclein interaction. ${ }^{31}$ However, aggregated SOD $1^{\mathrm{G} 93 \mathrm{~A}}$ was not able to increase SOD1 aggregation in our experiments. This finding contradicts results of other groups-even if they used a noncellular environment, different mutations, or fluorescent tags-who demonstrated the ability of misfolded SOD1 to seed its aggregation. ${ }^{13,14,32}$ This finding might be explained by the use of PCA detecting interaction of SOD1 and not its morphology as used by previous studies. ${ }^{13,14,32}$ Regarding our result, additional SOD1 possibly acts as competing counterpart for SOD1 protein complementation because the added SOD1 might form oligomers with the SOD1 labeled with the first half of the luciferase, thereby preventing it from joining the second half of the luciferase. In this way, the decreased luciferase activity in the presence of SOD1 ${ }^{\text {G93A }}$ aggregates can be explained. Because a direct interaction of $\alpha$-synuclein and SOD1 has been reported, ${ }^{31}$ $\alpha$-synuclein and SOD1-luciferase constructs potentially compete for the same binding side on the other SOD1luciferase half, which could lead to an underestimation of oligomer induction caused by $\alpha$-synuclein.

Consistent with the findings from PCA, we could also demonstrate an elevation of SOD $1^{\mathrm{G} 93 \mathrm{~A}}$ aggregate counts after intracerebral injection of $\alpha$-synuclein in vivo. Similar to the reported aggregation speed of $\alpha$-synuclein in wildtype mice, ${ }^{7}$ a higher accumulation rate of SOD $1^{\mathrm{G} 93 \mathrm{~A}}$ was observed $70 \mathrm{dpi}$ and not at earlier stages. We did not detect a significant difference at end stage of disease, which might derive from the clinical definition of end stage (ie, each individual has a maximal general pathologic degeneration covering the differences regarding higher aggregation rates observed in $\alpha$-synuclein PFF inoculated mice). Consistent with the proposed aggregation speed of $\alpha$-synuclein in wild-type mice, ${ }^{7} \alpha$-synuclein is elevated in $\alpha$-synuclein-injected animals at $70 \mathrm{dpi}$ as well. We could not demonstrate changes of survival, motor neuron counts. However, this might be caused by a fast disease progression of $\mathrm{SOD}^{G 93 A}$ mice. Possibly in a slower progressing mouse model with longer survival, motor neuron counts might also be influenced. In addition, additional studies should include methods that are able to detect functional differences between the
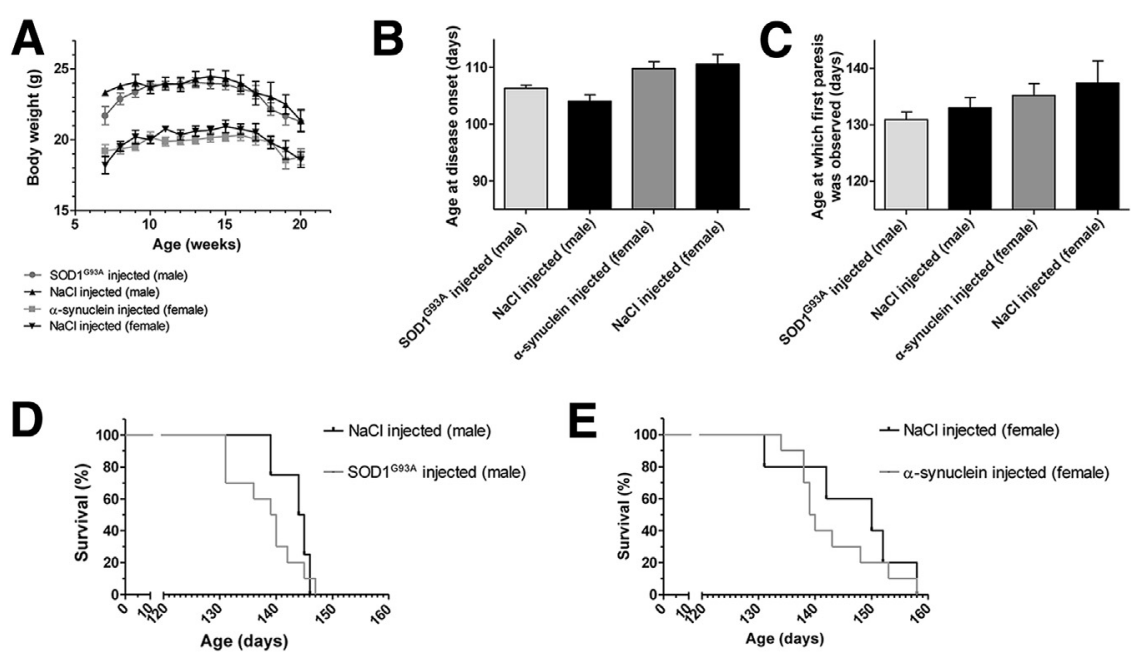

Figure 6 No changes in disease course of aggregate injected $S O D 1^{G 93 A}$ mice. A: No difference in body weight between the experimental groups is seen. B: There is no difference with age of mice at disease onset. C: There is no difference regarding the age when mice had first pareses. Kruskal-Wallis test and Dunns post test were performed (B and $\mathbf{C})$. D and E: A sex-specific difference but no difference between aggregate injected and corresponding controls is observed in the survival time. Data are expressed as means \pm SEM. $n=5 \mathrm{NaCl}$-injected mice (male and female; $\mathbf{A}, \mathbf{D}$, and $\mathbf{E}$ ); $n=10$ SOD $1^{\mathrm{G} 93 \mathrm{~A}}$ - and $\alpha$-synuclein-injected mice (A, D, and E); $n=5 \mathrm{NaCl}$-injected mice (male and female; C); $n=7 \mathrm{NaCl}$-injected mice (female; $\mathbf{B}$ ); $n=8 \mathrm{NaCl}-$ injected mice (male; B); $n=10 \mathrm{SOD}^{\mathrm{G} 93 \mathrm{~A}}$-injected mice (male) and $\alpha$-synuclein-injected mice (female; C); $n=13 \alpha$-synuclein-injected mice (female; B); $n=15$ SOD1 ${ }^{\text {G93A }}$-injected mice (male; B). 
differently inoculated mice (eg, motor unit number estimation).

Recently, another group found sparse SOD1 aggregation within the spinal cord 2.8 months after injection of mutated SOD1 and a general induction of motor neuron symptoms in heterozygous $S O D^{G 85 R-Y F P}$ mice. ${ }^{15}$ This model is normally asymptomatic and reveals no general pathologic accumulation. In contrast, the $S O D 1^{G 93 A}$ transgenic mice used here have an increasing load of SOD $1^{\mathrm{G} 93 \mathrm{~A}}$ accumulation in brainstem during the disease course. ${ }^{33,34}$ Therefore, it might not be surprising that no effect of SOD1 ${ }^{\mathrm{G} 93 \mathrm{~A}}$ aggregate inoculation on the total aggregate load in SOD1 $1^{G 93 A}$ transgenic mice could be observed because pathologic degeneration might cover the presence of aggregate formation processes. Consistent with our data, Ayers et $\mathrm{al}^{15}$ did not detect an accelerated disease progression in $S O D 1^{G 93 A}$ mice after SOD $1^{\mathrm{G} 93 \mathrm{~A}}$ inoculation.

In addition, evidence is emerging that different strains of SOD1 aggregates exist whose abundance correlates with disease progression in mice. ${ }^{35}$ Notably, only the less potent SOD1 strain could be isolated from SOD ${ }^{G 93 A}$ mice. ${ }^{35}$

Because the detailed mechanism of $\alpha$-synuclein and SOD1 interaction has not been characterized yet, we can only speculate on the influence of $\alpha$-synuclein on SOD1 ${ }^{\mathrm{G} 93 \mathrm{~A}}$ aggregation. On the one hand, $\alpha$-synuclein might act as a trigger for SOD1 ${ }^{\mathrm{G} 93 \mathrm{~A}}$ to form seeds that are further transported through neural networks and form aggregates only in susceptible areas. On the other hand, $\alpha$-synuclein itself could be transported and influence SOD1 ${ }^{\mathrm{G} 93 \mathrm{~A}}$ that is already in the aggregation process. In addition, the importance of a special cellular environment or regional susceptibility in the process of SOD1 ${ }^{\mathrm{G} 93 \mathrm{~A}}$ aggregation should be addressed in the future. However, we observed that the amount of $\alpha$-synuclein found in the facial nuclei is higher 70 dpi after injection of $\alpha$-synuclein PFFs compared with the injection of SOD1 ${ }^{\mathrm{G} 93 \mathrm{~A}}$ and $\mathrm{NaCl}$. This finding indicates that $\alpha$-synuclein levels influence the number of SOD1 aggregates directly because this measure is elevated in the same animals.

Regarding the transport of seeds, we hypothesize that they spread from insular cortex to the motor cortex. Both are interconnected according to tracing experiments. ${ }^{36,37}$ From the motor cortex, which is interconnected between both hemispheres through the corpus callosum, the corticospinal tract arises, projecting ipsilaterally and contralaterally to the motoric areas of the brainstem. ${ }^{38}$ In addition, projections exist from the motor cortex to relay neurons located in the brainstem. ${ }^{39}$ Therefore, we can state that the injection site is connected to the facial nucleus by polysynaptic connections, suggesting a route of transport. However, this route of seed transportation should be validated using tracers in further experiments.

Taken together, this study indicates for the first time that $\alpha$-synuclein PFFs increase SOD1 aggregation in vitro and in vivo. The underlying mechanism seems to enhance selfaggregation of wild-type and mutant SOD1. Suppressing such an influence might be an additional therapeutic target in ALS. Therefore, in future studies, cofactors in the aggregation process should be investigated.

\section{Acknowledgments}

We thank Stephen Meier for performing large parts of intracerebral injections, Evamaria Görz for technical assistance, Marisa Feiler for cloning L1 and L2 constructs, and Axel Freischmidt for generating SOD1-L1 and -L2 plasmids (all from the Department of Neurology, University of Ulm).

\section{Supplemental Data}

Supplemental material for this article can be found at http://dx.doi.org/10.1016/j.ajpath.2016.04.008.

\section{References}

1. Jellinger KA: Recent advances in our understanding of neurodegeneration. J Neural Transm 2009, 119:1111-1162

2. Costanzo M, Zurzolo C: The cell biology of prion-like spread of protein aggregates: mechanisms and implication in neurodegeneration. Biochem J 2013, 452:1-17

3. Grad LI, Cashman NR: Prion-like activity of $\mathrm{Cu} / \mathrm{Zn}$ superoxide dismutase. Prion 2014, 8:33-41

4. Braak H, Del Tredici K, Rüb U, De Vos RAI, Jansen Steur EN, Braak E: Staging of brain pathology related to sporadic Parkinson's disease. Neurobiol Aging 2003, 24:197-211

5. Braak H, Braak E: Neuropathological stageing of Alzheimer-related changes. Acta Neuropathol 1991, 82:239-259

6. Weissmann C: A "unified theory" of prion propagation. Nature 1991, 352:679-683

7. Masuda-Suzukake M, Nonaka T, Hosokawa M, Kubo M, Shimozawa A, Akiyama H, Hasegawa M: Pathological alphasynuclein propagates through neural networks. Acta Neuropathol Commun 2014, 2:88

8. Rangel A, Race B, Phillips K, Striebel J, Kurtz N, Chesebro B: Distinct patterns of spread of prion infection in brains of mice expressing anchorless or anchored forms of prion protein. Acta Neuropathol Commun 2014, 2:8

9. Stancu IC, Vasconcelos B, Ris L, Wang P, Villers A, Peeraer E, Buist A, Terwel D, Baatsen P, Oyelami T, Pierrot N, Casteels C, Bormans G, Kienlen-Campard P, Octave JN, Moechars D, Dewachter I: Templated misfolding of Tau by prion-like seeding along neuronal connections impairs neuronal network function and associated behavioral outcomes in Tau transgenic mice. Acta Neuropathol 2015, 129:875-894

10. Nath S, Agholme L, Kurudenkandy FR, Granseth B, Marcusson J, Hallbeck M: Spreading of neurodegenerative pathology via neuron-toneuron transmission of -amyloid. J Neurosci 2012, 32:8767-8777

11. Lee S, Kim HJ: Prion-like mechanism in amyotrophic lateral sclerosis: are protein aggregates the key? Exp Neurobiol 2015, 24:1

12. Gurney ME: Transgenic-mouse model of amyotrophic lateral sclerosis N Engl J Med 1994, 331:1721-1722

13. Münch C, O'Brien J, Bertolotti A: Prion-like propagation of mutant superoxide dismutase-1 misfolding in neuronal cells. Proc Natl Acad Sci U S A 2011, 108:3548-3553

14. Grad LI, Guest WC, Yanai A, Pokrishevsky E, O’Neill MA, Gibbs E, Semenchenko V, Yousefi M, Wishart DS, Plotkin SS, Cashman NR: Intermolecular transmission of superoxide dismutase 1 misfolding in living cells. Proc Natl Acad Sci 2011, 108:16398-16403 
15. Ayers JI, Fromholt S, Koch M, DeBosier A, McMahon B, Xu G, Borchelt DR: Experimental transmissibility of mutant SOD1 motor neuron disease. Acta Neuropathol 2014, 128:791-803

16. Spillantini MG, Schmidt ML, Lee VM, Trojanowski JQ, Jakes R, Goedert M: Alpha-synuclein in Lewy bodies. Nature 1997, 388:839-840

17. Takei YI, Oguchi K, Koshihara H, Hineno A, Nakamura A, Ohara S: $\alpha$-Synuclein coaggregation in familial amyotrophic lateral sclerosis with SOD1 gene mutation. Hum Pathol 2013, 44:1171-1176

18. Gurney ME, Pu H, Chiu AY, Dal Canto MC, Polchow CY, Alexander DD, Caliendo J, Hentati A, Kwon YW, Deng HX: Motor neuron degeneration in mice that express a human $\mathrm{Cu}, \mathrm{Zn}$ superoxide dismutase mutation. Science 1994, 264:1772-1775

19. Luk KC, Song C, O'Brien P, Stieber A, Branch JR, Brunden KR, Trojanowski JQ, Lee VM: Exogenous alpha-synuclein fibrils seed the formation of Lewy body-like intracellular inclusions in cultured cells. Proc Natl Acad Sci U S A 2009, 106:20051-20056

20. Liebl MP, Kaya AM, Tenzer S, Mittenzwei R, Koziollek-Drechsler I, Schild H, Moosmann B, Behl C, Clement AM: Dimerization of visinin-like protein 1 is regulated by oxidative stress and calcium and is a pathological hallmark of amyotrophic lateral sclerosis. Free Radic Biol Med 2014, 72:41-54

21. Kramer ML, Schulz-Schaeffer WJ: Presynaptic alpha-synuclein aggregates, not Lewy bodies, cause neurodegeneration in dementia with Lewy bodies. J Neurosci 2007, 27:1405-1410

22. Wang J, Xu G, Borchelt DR: High molecular weight complexes of mutant superoxide dismutase 1: age-dependent and tissue-specific accumulation. Neurobiol Dis 2002, 9:139-148

23. Danzer KM, Ruf WP, Putcha P, Joyner D, Hashimoto T, Glabe C, Hyman BT, McLean PJ: Heat-shock protein 70 modulates toxic extracellular $\alpha$-synuclein oligomers and rescues trans-synaptic toxicity. FASEB J 2011, 25:326-336

24. Steinacker P, Hawlik A, Lehnert S, Jahn O, Meier S, Görz E, Braunstein KE, Krzovska M, Schwalenstöcker B, Jesse S, Pröpper C, Böckers T, Ludolph A, Otto M: Neuroprotective function of cellular prion protein in a mouse model of amyotrophic lateral sclerosis. Am J Pathol 2010, 176:1409-1420

25. Alexander GM, Erwin KL, Byers N, Deitch JS, Augelli BJ, Blankenhorn EP, Heiman-Patterson TD: Effect of transgene copy number on survival in the G93A SOD1 transgenic mouse model of ALS. Brain Res Mol Brain Res 2004, 130:7-15

26. Putcha P, Danzer KM, Kranich LR, Scott A, Silinski M, Mabbett S, Hicks CD, Veal JM, Steed PM, Hyman BT, McLean PJ: Brainpermeable small-molecule inhibitors of Hsp90 prevent alpha-synuclein oligomer formation and rescue alpha-synuclein-induced toxicity. J Pharmacol Exp Ther 2010, 332:849-857

27. Luk KC, Kehm V, Carroll J, Zhang B, Brien PO, Trojanowski JQ, Lee VM: Pathological alpha-synuclein transmission initiares parkinson-like neurodegeneration in nontransgenic mice. Science 2012, 338:949-953
28. Beekes M, Thomzig A, Schulz-Schaeffer WJ, Burger R: Is there a risk of prion-like disease transmission by Alzheimer- or Parkinson-associated protein particles? Acta Neuropathol 2014, 128:463-476

29. Planchard MS, Exley SE, Morgan SE, Rangachari V: Dopamineinduced $\alpha$-synuclein oligomers show self- and cross-propagation properties. Protein Sci 2014, 10:1369-1379

30. Urushitani M, Sik A, Sakurai T, Nukina N, Takahashi R, Julien JP: Chromogranin-mediated secretion of mutant superoxide dismutase proteins linked to amyotrophic lateral sclerosis. Nat Neurosci 2006, 9: $108-118$

31. Helferich AM, Ruf WP, Grozdanov V, Freischmidt A, Feiler MS, Zondler L, Ludolph AC, McLean PJ, Weishaupt JH, Danzer KM: $\alpha$-Synuclein interacts with SOD1 and promotes its oligomerization. Mol Neurodegener 2015, 10:66

32. Chia R, Tattum MH, Jones S, Collinge J, Fisher EM, Jackson GS: Superoxide dismutase 1 and tgSOD1 mouse spinal cord seed fibrils, suggesting a propagative cell death mechanism in amyotrophic lateral sclerosis. PLoS One 2010, 5:e10627

33. An T, Shi P, Duan W, Zhang S, Yuan P, Li Z, Wu D, Xu Z, Li C, Guo Y: Oxidative stress and autophagic alteration in brainstem of SOD1-G93A mouse model of ALS. Mol Neurobiol 2014, 49: $1435-1448$

34. Steinacker P, Berner C, Thal DR, Attems J, Ludolph AC, Otto M: Protease-resistant SOD1 aggregates in amyotrophic lateral sclerosis demonstrated by paraffin-embedded tissue (PET) blot. Acta Neuropathol Commun 2014, 2:130

35. Bergh J, Zetterström $\mathrm{P}$, Andersen PM, Brännström T, Graffmo KS, Jonsson PA, Lang L, Danielsson J, Oliveberg M, Marklund SL: Structural and kinetic analysis of protein-aggregate strains in vivo using binary epitope mapping. Proc Natl Acad Sci U S A 2015, 112: 4489-4494

36. Shi CJ, Cassell MD: Cortical, thalamic, and amygdaloid connections of the anterior and posterior insular cortices. J Comp Neurol 1998, 399: 440-468

37. Oh SW, Harris JA, Ng L, Winslow B, Cain N, Mihalas S, Wang Q, Lau C, Kuan L, Henry AM, Mortrud MT, Ouellette B, Nguyen TN, Sorensen SA, Slaughterbeck CR, Wakeman W, Li Y, Feng D, Ho A, Nicholas E, Hirokawa KE, Bohn P, Joines KM, Peng H, Hawrylycz MJ, Phillips JW, Hohmann JG, Wohnoutka P, Gerfen CR, Koch C, Bernard A, Dang C, Jones AR, Zeng H: A mesoscale connectome of the mouse brain. Nature 2014, 508: 207-214

38. Carmel JB, Martin JH: Motor cortex electrical stimulation augments sprouting of the corticospinal tract and promotes recovery of motor function. Front Integr Neurosci 2014, 8:51

39. Jankowska E, Edgley SA: How can corticospinal tract neurons contribute to ipsilateral movements? A question with implications for recovery of motor functions. Neuroscientist 2006, 12:67-79 